\title{
A New Approach to Predicting Postconcussion Syndrome after Mild Traumatic Brain Injury based upon Eye Movement Function
}

\author{
Marcus H. Heitger, Richard D. Jones, Senior Member, IEEE, Tim J. Anderson
}

\begin{abstract}
Following on from our earlier findings of a close relationship between motor function and outcome after mild traumatic brain injury (mTBI), this study examined whether it might be possible to predict poor recovery in the form of postconcussion syndrome (PCS) based upon early eye and arm motor function. Within 1 week post-injury, we assessed 37 mTBI patients on measures of saccades, oculomotor smooth pursuit, upper-limb visuomotor function, neuropsychological status, and self-reported health condition. At 3 months, 8 patients met the criteria for PCS. Using discriminant function analyses, we examined whether this future PCS-group could be identified prospectively based on motor function, neuropsychological status, and self-reported health condition at 1 week post-injury. Early eye movement function was the most effective in distinguishing between PCS and non-PCS patients, achieving a sensitivity and specificity of $100 \%$ in the present sample. This was followed by self-reported early health condition (sensitivity: $87 \%$, specificity: 97\%), early upper-limb motor performance $(87 \%, 97 \%)$, neuropsychological function $(62 \%, 100 \%)$, and age, gender, education and clinical measures of trauma severity $(37 \%, 87 \%)$. Leave-one-out validation analyses confirmed eye movements as the most robust discriminator (sensitivity: 62\%, specificity: 97\%). Assessment of eye movement function after mTBI may contribute to a prospective identification of patients who develop PCS, supporting the targeting of early health-care intervention.
\end{abstract}

\section{INTRODUCTION}

$\mathrm{T}$ HE occurrence of post-concussion syndrome (PCS) after mild traumatic brain injury (mTBI) is one of the most puzzling phenomena in the context of head trauma. This syndrome can affect up to $20-30 \%$ of mTBI patients and comprises incomplete recovery and persistence of

Manuscript received April 16, 2008. The study was hosted by the Canterbury District Health Board and the University of Otago Christchurch, New Zealand. Funding for patients' travel costs was provided by the Neurological Foundation of New Zealand (grant number 0027/SPG).

M. H. Heitger is a Research Fellow in the Dept. of Medicine at the University of Otago - Christchurch, New Zealand, and is based at the Van der Veer Institute for Parkinson's \& Brain Research in Christchurch (phone: +64 3 3786074; fax: +64 3378 6080; marcus.heitger@otago.ac.nz).

R. D. Jones is a senior biomedical engineer \& neuroscientist in the Dept. of Medical Physics and Bio-engineering, Christchurch Hospital, Christchurch, New Zealand, a Research Associate Professor in the Dept. of Medicine, University of Otago - Christchurch, and an Associate Professor in the Dept. of Electrical \& Computer Engineering at the University of Canterbury, Christchurch (richard.jones@otago.ac.nz).

T. J. Anderson holds the Cas Van der Veer chair in Parkinson's Disease and Movement Disorders at the University of Otago - Christchurch, New Zealand, and is the Research Director of the Parkinson's Disease and Related Disorders Division at the Van der Veer Institute for Parkinson's \& Brain Research (tim.anderson@otago.ac.nz). postconcussional symptoms for weeks, months or even years post-injury, having a potentially devastating effect on a patient's life [1,2]. PCS is recognized as a clinical entity by the World Health Organization [3]. However, one of the major problems with PCS is that it is very difficult to tell early post-injury which patients will develop PCS. Previous studies investigating clinical measures of injury severity, early symptom status, biochemical markers, neuropsychological function, and brain imaging have had unsatisfactory results in identifying predictors of PCS [4,5].

We have previously reported that mTBI impairs the complex cerebral networks for eye and arm motor control $[4,6]$. These motor deficits are independent of intellectual ability and occur independently of neuropsychological impairment after mTBI [4]. The composition of impaired and preserved motor functions indicated that motor function relates closely to the functional status of the brain after mTBI [4,6]. Using a multiple regression approach, we identified a systematic group-level relationship between motor-function at approximately one week post-injury and level of recovery at 3 and 6 months [5]. In the present paper, we explore whether this relationship between early motor function and recovery might be useful in the early identification of patients who will develop PCS, and how motor function compares to self-reported health condition and early neuropsychological status in its ability to predict the development of PCS.

\section{METHODS}

\section{A. Participants}

Thirty seven subjects (13 female and 24 male) with mTBI (Glasgow Coma Scale score 13: 4 cases, 14: 12 cases, 15: 21 cases) were recruited from patients presenting with acute head injury to Christchurch Hospital (the principal hospital for a population pool $>400,000)$. All had experienced posttraumatic amnesia (PTA) (median $15 \mathrm{~min}$, range $2 \mathrm{~min}-22$ h) and 32 had a confirmed loss of consciousness (LOC) (median $2.0 \mathrm{~min}$, range $0.5-15 \mathrm{~min}$ ). Mean age was 29.1 years (SD 12.7, range 15-56 years) and mean years of education 13.6 (SD 2.56, range 8-19 years). All patients were employed or attending institutions for secondary or tertiary education, and none was involved in litigation. Other potential participants were excluded if there was evidence of any influence of alcohol or psychoactive drugs at time of injury, regular intake of psychoactive drugs or history of 
drug abuse, central neurological disorder or psychiatric condition, structural brain damage or haematoma on CT head scan, skull fractures, or prior history of head injury with persisting symptoms or complaints.

Patients were assessed within one week of injury (mean $5.5 \pm$ SD 3.0 days) and then at 3 months (90 \pm 5.5 days). Throughout the study, none of the participants were hospitalized or developed health problems related to other causes which could have affected any of the measures. The project was approved by the Canterbury Ethics Committee and written consent was obtained from all participants.

\section{B. Assessments}

1) Oculomotor testing: The methods, paradigm parameters, and key measures have been described in detail elsewhere [4,6]. We incorporated reflexive saccades, antisaccades, memory-guided sequences of saccades, selfpaced saccades, and sine and random oculomotor smooth pursuit. Key measures were saccade latency (ms) (reflexive, anti- and self-paced saccades), saccade velocity ( $\%$ s) (reflexive, anti- and self-paced saccades), number of selfpaced saccades within 30s, directional errors (antisaccades and memory-guided sequences), and, for all saccadic paradigms, the mean absolute position error of the final eye position $(\mathrm{PE}=\mid($ EPfin $-\mathrm{SP}) / \mathrm{SP} \mid * 100)$, and gain of the primary saccade (EPprim / SP) and final eye position (EPfin / SP), where EPprim is the eye position after the initial saccade, EPfin is the final eye position and SP is the stimulus position. For memory-guided sequences, an amplitude error (AE) was also derived for all sequence steps. The AE is based on movement size rather than the absolute size of error between final fixation and stimulus position $(\mathrm{AE}=$ mean difference [\%] between observed saccadic vs. stimulus amplitude). An absolute time index (subject's total response time/duration of the sequence) was also calculated for memory-guided sequences. Key measures for oculomotor smooth pursuit were the average eye peak velocity $(\%)$ after removal of all saccades from the tracking performance and the tracking lag (ms). Overall, the oculomotor assessment provided 35 variables for the discriminant function analyses.

2) Upper-limb visuomotor testing: The equipment, paradigm parameters, and key measures were identical to a previous study [4], comprising tests of visual acuity, visual perception and resolution, arm movement speed and steadiness, and several one-dimensional (1D) visuomotor tracking tasks (sine and random preview/non-preview and step tracking) [4]. The upper-limb visuomotor tests provided 13 variables for the discriminant function analyses.

3) Neuropsychological tests: Attention, working memory, episodic memory, and speed of information processing were assessed by the Paced Auditory Serial Addition Task [7], California Verbal Learning Test I [8], Symbol Digit Modalities Test [9], and Trail Making Test A+B [10]. General cognitive performance was evaluated with the Vocabulary Test and Matrix Reasoning subtests of the
Wechsler Abbreviated Scale of Intelligence [11]. Standardized instructions were followed for all tests. The neuropsychological measures provided 27 variables for the discriminant function analyses.

4) Health status measures: The study incorporated several health assessment measures to quantify the subjects' current health condition, level of postconcussional complaints, and impact of mTBI on performance of everyday tasks. These measures were the SF-36 Health Survey (version 2) [12], the Rivermead Postconcussion Symptoms Questionnaire (RPSQ) [13], and the Rivermead Head Injury Follow-up Questionnaire (RHIFQ) [14]. Subjects had to answer the written questions in the presence of the examiner. Details of this assessment have been described previously [5,6]. The three health status questionnaires provided 38 variables for the discriminant function analyses.

\section{Classification of postconcussion syndrome}

The decision on whether a patient qualified for the diagnosis of PCS at 3 months post-injury was based on the diagnostic criteria of the WHO 10th International Classification of Diseases [3]. The assessment on the ICD-10 criteria was primarily based on the patients' scores on the RPSQ covering the symptom categories 1-4. Patients had to have experienced a LOC and had to show symptoms in at least 3 symptom categories of the WHO criteria. Patients were not rated on symptom category 5 'reduced alcohol tolerance' as many of the patients did not have a regular alcohol intake or were under the legal drinking age of 18 years. The SF-36 General Health scale served as a surrogate measure for category 6 'preoccupation with present symptoms, adoption of sick role and fear of permanent brain damage'. Yet, assessment on the SF-36 did not change the Rivermead-based PCS classification in any of the patients.

\section{Statistical analysis}

Stepwise-forward discriminant function analysis was applied to formulate statistical models able to discriminate between prospective PCS and non-PCS patients at 1 week post-injury. The 'F-to-enter' was 1.0 and 'F-to-remove' 0.5 . Independent variables were selected from the measure categories (i.e., oculomotor, upper-limb visuomotor, neuropsychological, health status, and clinical severity measures together with age, gender and education) to discern the specific potential of any of these modalities to distinguish between prospective PCS and non-PCS patients at 1 week post-injury. The maximum number of in-model variables during the discriminant function analyses was limited to 10 variables. A further limitation was not considered necessary as it was not the primary aim of this analysis to create models unequivocally applicable to any new patient set but to compare the relative capacity of different modalities at 1 week to discriminate between the PCS and non-PCS patients in the current data set. Discriminatory power of the models was quantified by the resultant sensitivity, specificity, and the Wilks' lambda statistic $(1.0=$ no discriminatory power; 
$0.0=$ perfect discriminatory power). In order to estimate whether the discriminative power achieved in the present sample of 37 patients would be achieved in separate but similar mTBI groups, we conducted a leave-one-out analysis for the main modalities. For this analysis, discriminant models were calculated following the same principles as in the complete patient sample but only including 36 cases in the training set whilst 1 case was left aside. The data from this case was then used as 'external data' to test if the model recognized the case correctly as PCS or non-PCS. This was done 37 times in each modality so that each patient was the external case at one stage. Key measures (in \%) of this leaveone-out process were overall classification accuracy (number of patients correctly classified when they were the left-outcase / 37), sensitivity (number of PCS-cases correctly recognized / 8), and specificity (number of non-PCS cases correctly recognized / 29). In addition, we calculated the False-positive-rate (number of non-PCS patients incorrectly identified as 'PCS' / cases classified as 'PCS') and Falsenegative-rate (number of PCS patients incorrectly identified as 'non-PCS' / cases classified as 'non-PCS').

\section{RESULTS}

\section{A. Study groups}

Eight patients $(21 \%)$ met the PCS criteria at 3 months post-injury. The PCS and non-PCS groups differed markedly in health status, with the PCS group having poorer scores on RPSQ-total (PCS-group mean \pm SD: $24.6 \pm 8.9$ vs. non-PCS: $5.7 \pm 5.1, p<0.0001)$, RHIFQ-total $(6.4 \pm 6.9$ vs. $1.5 \pm 1.9$, $p=0.004)$ and SF-36 Mental Health scale $(65.0 \pm 11.0$ vs. $78.5 \pm 12.0, p=0.014$ ), plus a marginal difference on the SF-36 Vitality scale $(54.7 \pm 6.5$ vs. $64.0 \pm 15.5, p=0.06)$. There was no difference between the two groups in terms of severity of clinical measures at 1 week post-injury, age, gender, and years of education (Table 1).

TABLE I

PCS VS NON-PCS PATIENTS - INJURY SEVERITY AND PREMORBID FACTORS

\begin{tabular}{|c|c|c|c|c|c|}
\hline & \multicolumn{2}{|c|}{$\operatorname{PCS}(n=8)$} & \multicolumn{2}{|c|}{ Non-PCS $(n=29)$} & \multirow[b]{2}{*}{$p$-level } \\
\hline & Mean & SD & Mean & SD & \\
\hline Glasgow Coma Scale & 14.4 & 0.7 & 14.5 & 0.7 & 0.71 \\
\hline $\begin{array}{r}\text { Mean PTA duration (min) } \\
\text { Median (quartiles) }\end{array}$ & $\begin{array}{r}89.4 \\
10(7.5 / 67.5)\end{array}$ & 186.2 & $\begin{array}{r}178.1 \\
20(5.0 / 210)\end{array}$ & 345.8 & 0.54 \\
\hline $\begin{array}{r}\text { Mean LOC (min) } \\
\text { Median (quartiles) }\end{array}$ & $\begin{array}{c}2.3 \\
1.5(1.0 / 3.5)\end{array}$ & 1.8 & $\begin{array}{r}4.7 \\
2.0(1.0 / 7.5)\end{array}$ & 4.7 & 0.24 \\
\hline Mean age & 24.5 & 12.1 & 30.4 & 12.8 & 0.22 \\
\hline Gender (F/M, \%) & $37.5 / 62.5$ & & $34.5 / 65.5$ & & \\
\hline Mean years of education & 12.6 & 1.5 & 13.9 & 2.7 & 0.22 \\
\hline First assessment (days) & 5.1 & 3.2 & 5.6 & 3.0 & 0.66 \\
\hline
\end{tabular}

\section{B. Discriminant function analysis}

The model based exclusively on measures of saccades and oculomotor smooth pursuit was able to detect all prospective PCS patients, while classifying none of the remaining patients as 'false-positives' (Table 2). The model based on the combined application of eye and upper-limb motor measures showed similar results (Table 2). While none of these models reached a Wilks' lambda of zero, their discriminatory power was sufficient to correctly classify all patients in the current TBI group as prospective PCS or nonPCS candidates. The model based on measures of health status at 1 week took 'third place', with an overall discriminatory power slightly weaker than observed for the eye / eye+arm models, together with poorer sensitivity, specificity and detection scores (Table 2). The discriminatory power of early neuropsychological status was considerably lower, which equally applied to measures of upper-limb visuomotor function if used in isolation. Both models generally showed poorer sensitivity and specificity, although the neuropsychological model was able to reach a $100 \%$ specificity (i.e., no 'false-positive' detections amongst the non-PCS patients). The smallest discriminatory power was observed for measures of injury severity, age, gender, and years of education.

TABLE II

PCS VS NON-PCS DISCRIMINANT FUNCTION ANALYSIS POWER OF DIFFERENT MODELS

\begin{tabular}{|c|c|c|c|c|c|}
\hline $\begin{array}{l}\text { Independent } \\
\text { measure pool }\end{array}$ & $\begin{array}{l}\text { Wilks' } \\
\text { Lambda }\end{array}$ & $p$-level & $\begin{array}{l}\text { Variables } \\
\text { in model }\end{array}$ & Classifications & \\
\hline $\begin{array}{l}\text { Eye movement } \\
\text { measures }\end{array}$ & 0.18 & $<0.001$ & 10 & $\begin{array}{l}\text { Sensitivity (\%) } \\
\text { Specificity (\%) } \\
\text { False-negatives (\%) } \\
\text { False-positives (\%) }\end{array}$ & $\begin{array}{c}100 \\
100 \\
0 \\
0\end{array}$ \\
\hline $\begin{array}{l}\text { Upper-limb } \\
\text { visuomotor } \\
\text { function }\end{array}$ & 0.49 & 0.002 & 7 & $\begin{array}{l}\text { Sensitivity (\%) } \\
\text { Specificity (\%) } \\
\text { False-negatives (\%) } \\
\text { False-positives (\%) }\end{array}$ & $\begin{array}{c}87.5 \\
96.6 \\
3.4 \\
12.5\end{array}$ \\
\hline $\begin{array}{l}\text { Eye \& Arm motor } \\
\text { measures } \\
\text { combined }\end{array}$ & 0.19 & $<0.001$ & 10 & $\begin{array}{l}\text { Sensitivity (\%) } \\
\text { Specificity (\%) } \\
\text { False-negatives (\%) } \\
\text { False-positives (\%) }\end{array}$ & $\begin{array}{c}100 \\
100 \\
0 \\
0\end{array}$ \\
\hline $\begin{array}{c}\text { Neuro- } \\
\text { psychological } \\
\text { measures }\end{array}$ & 0.61 & 0.02 & 6 & $\begin{array}{l}\text { Sensitivity (\%) } \\
\text { Specificity (\%) } \\
\text { False-negatives (\%) } \\
\text { False-positives (\%) }\end{array}$ & $\begin{array}{l}62.5 \\
100 \\
9.4 \\
0.0\end{array}$ \\
\hline $\begin{array}{l}\text { Self-reported } \\
\text { health status }\end{array}$ & 0.25 & $<0.001$ & 10 & $\begin{array}{l}\text { Sensitivity (\%) } \\
\text { Specificity (\%) } \\
\text { False-negatives (\%) } \\
\text { False-positives (\%) }\end{array}$ & $\begin{array}{c}87.5 \\
96.6 \\
3.4 \\
12.5\end{array}$ \\
\hline $\begin{array}{l}\text { GCS, LOC, PTA, } \\
\text { age, gender, } \\
\text { education }\end{array}$ & 0.79 & 0.09 & 3 & $\begin{array}{l}\text { Sensitivity (\%) } \\
\text { Specificity (\%) } \\
\text { False-negatives (\%) } \\
\text { False-positives (\%) }\end{array}$ & $\begin{array}{l}37.5 \\
87.0 \\
20.0 \\
50.0 \\
\end{array}$ \\
\hline
\end{tabular}

\section{Leave-one-out analysis}

In all modalities, sensitivity and specificity decreased in the leave-one-out analysis compared to the discriminative efficiency achieved in the initial training set (Table 3). Eye movement function had the smallest 'ground loss' and was superior to combined eye and arm motor function, selfreported health status and neuropsychological function in classifying prospective PCS and non-PCS cases. Interestingly, neuropsychological function had better results than self-reported health status, which had the poorest sensitivity and specificity (Table 3 ). 
TABLE III

LEAVE-ONE-OUT ANALYSIS - DISCRIMINATORY POWER OF DIFFERENT MODALITIES

\begin{tabular}{clc}
\hline Independent measure pool & Classifications & \\
\hline Eye movement measures & Overall accuracy (\%) & 89.2 \\
& Sensitivity (\%) & 62.5 \\
& Specificity (\%) & 96.6 \\
& False-negatives (\%) & 9.7 \\
Eye and arm motor & False-positives (\%) & 16.7 \\
measures combined & Overall accuracy (\%) & 86.5 \\
& Sensitivity (\%) & 50 \\
& Specificity (\%) & 96.6 \\
& False-negatives (\%) & 12.5 \\
Neuropsychological & False-positives (\%) & 20 \\
measures & Overall accuracy (\%) & 86.5 \\
& Sensitivity (\%) & 62.5 \\
& Specificity (\%) & 93.1 \\
& False-negatives (\%) & 10 \\
& False-positives (\%) & 28.6 \\
Self-reported & Overall accuracy (\%) & 67.6 \\
health status measures & Sensitivity (\%) & 12.5 \\
& Specificity (\%) & 82.8 \\
& False-negatives (\%) & 22.6 \\
& False-positives (\%) & 83.3 \\
\hline
\end{tabular}

\section{DISCUSSION}

Our findings suggest that eye movement function might make useful contributions to an early identification of patients likely to develop PCS after mTBI. Eye movement function had better results than observed for upper-limb visuomotor measures. This is likely due to the inclusion of a wider range of functional measures in the eye movement assessment, therefore capturing a wider range of brain functions. The poor performance of the neuropsychological models is consistent with previous findings that neuropsychological assessment provides unsatisfactory results in gauging future brain function after mTBI $[1,2,4,6]$. The discrimination model based on the Rivermead questionnaires and the SF-36 showed the 'third-best' discriminatory power in the training set. This indicates that evaluation of early health status has some predictive properties, the 'set-back' being that it is based on subjective patient self-report. However, the much poor results of the health status measures in the leave-one-out analysis show that it is difficult to reliably transfer the relationship between idiosyncratic self-report of early postconcussional symptoms and respective recovery in 'historic' cases of mTBI to 'the next case'. The model based on measures of injury severity, age, gender, and years of education showed only a poor ability to identify prospective PCS cases. Naturally, the small number of variables in this model and its poor discriminatory power was facilitated by the smaller pool of measures to choose from. However, the current results indicate that there are no strong predictor variables for the later incidence of PCS amongst these variables. These results mirror earlier studies $[1,2]$. The discrimination models with eye movement contribution reached a Wilks' lambda $<0.2$, demonstrated perfect patient classifications in the current group, and were paired with strong levels of significance. This suggests that these findings likely reflect an effect present in the general
mTBI population. However, the leave-one-out analysis indicates that the predictive accuracy of this method will decrease substantially when applied to independent mTBI patients. This notwithstanding, eye movements remained as the most robust domain with regard to predictive accuracy. Whilst the corresponding sensitivity was poor $(62.5 \%)$, its high specificity warrants the expectation that, although some PCS cases will be missed, any positive detections are likely to face a slow recovery from mTBI. A prospective validation of this approach in an independent test set is essential to ascertain its general applicability after mTBI.

\section{CONCLUSION}

Our findings suggest that oculomotor assessment may contribute to predicting adverse outcome in the form of PCS after mTBI, thus providing a potential screening instrument with utility in a clinical context.

\section{REFERENCES}

[1] G. L. Iverson, "Outcome from mild traumatic brain injury", Curr. Opin. Psychiatry, vol. 18, pp. 301-317, 2005.

[2] L. J. Carroll, J. D. Cassidy, P. M. Peloso, J. Borg, H. von Holst, L. Holm et al., "Prognosis for mild traumatic brain injury: results of the WHO Collaborating Centre Task Force on Mild Traumatic Brain Injury", J. Rehabil. Med., vol. 43, pp. 84-105, 2004.

[3] The World Health Organization, "The ICD-10 Classification of Mental and Behavioural Disorders", 1992, WHO, Geneva, Switzerland.

[4] M. H. Heitger, T. J. Anderson, R. D. Jones, J. C. Dalrymple-Alford, C. M. Frampton and M. W. Ardagh, "Eye movement and visuomotor arm movement deficits following mild closed head injury", Brain, vol. 127, pp. 575-90, 2004.

[5] M. H. Heitger, R. D. Jones, J. C. Dalrymple-Alford, C. M. Frampton, M. W. Ardagh and T. J. Anderson, "Mild head injury - a close relationship between motor function at one week post-injury and overall recovery at three and six months", J. Neurol. Sci., vol. 253, pp. 34-47, 2007.

[6] M. H. Heitger, R. D. Jones, J. C. Dalrymple-Alford, C. M. Frampton, M. W. Ardagh and T. J. Anderson, "Motor deficits and recovery during the first year following mild closed head injury", Brain Inj., vol. 20, pp. 807-824, 2006.

[7] D. M. A. Gronwall, "Paced Auditory Serial Addition Test (PASAT)", 1977, Department of Psychology, University of Victoria, Victoria, Canada.

[8] D. C. Delis, Kramer, J.H., Kaplan, E., Ober, B.A., "California Verbal Learning Test (CVLT)", 1987, The Psychological Corporation®, Harcourt Brace \& Company, San Antonio.

[9] A. Smith, "Symbol Digit Modalities Test", 1973, Western Psychological Services, Los Angeles, USA, Los Angeles, California.

[10] O. Spreen and E. Strauss, "A compendium of neuropsychological tests", 1991, Oxford University Press, New York.

[11] The Psychological Corporation, "Wechsler Abbreviated Scale of Intelligence - Manual", 1999, The Psychological Corporation, San Antonio.

[12] J. E. Ware, M. Kosinski and J. E. Dewey, "How to Score Version Two of the SF-36 Health Survey", 2000, QualityMetric Incorporated, Lincoln, RI.

[13] N. S. King, S. Crawford, F. J. Wenden, N. E. Moss and D. T. Wade, "The Rivermead Post Concussion Symptoms Questionnaire: a measure of symptoms commonly experienced after head injury and its reliability", J. Neurol., vol. 242, pp. 587-92, 1995.

[14] S. Crawford, F. J. Wenden and D. T. Wade, "The Rivermead head injury follow up questionnaire: a study of a new rating scale and other measures to evaluate outcome after head injury", J. Neurol. Neurosurg. Psychiatry, vol. 60, pp. 510-4, 1996. 